Received: 09.01.2018

Revised: 26.01 .2018

Accepted: 16.02 .2018

DOI: $10.17804 / 2410-9908.2018 .1 .016-033$

\title{
ANALYSIS OF THE STRENGTH OF SHELL STRUCTURES, MADE FROM MODERN MATERIALS, ACCORDING TO VARIOUS STRENGTH CRITERIA
}

\author{
A. A. Semenov \\ Saint-Petersburg State University of Architecture and Civil Engineering, \\ 4, 2-ya Krasnoarmeyskaya st., Saint-Petersburg, 190005, Russian Federation \\ ه sw.semenov@gmail.com; D https://orcid.org/0000-0001-9490-7364 \\ Corresponding author. E-mail: sw.semenov@gmail.com \\ Address for correspondence: ul. 2-ya Krasnoarmeyskaya, 4, 190005, Saint-Petersburg, Russian Federation
}

The paper analyzes the possibility of applying five different strength criteria (maximum stress criterion, Mises-Hill, Pisarenko-Lebedev, Fisher, Goldenblat-Kopnov) to calculating the strength of orthotropic shell structures. We consider shallow shells of double curvature, square in plan, panels of cylindrical and conical shells. A geometrically nonlinear mathematical model of their deformation, taking into account transverse shearing, is used. For calculations, the characteristics of modern orthotropic materials are used, such as fiberglass and CFRP. An increase in the areas of the failure of strength conditions with increasing load is shown.

Keywords: strength, strength criterion, theory of strength, shell, CFRP.

\section{Funding}

The study was supported by the RF Ministry of Education and Science within the framework of the state order, project No. 9.5605.2017/8.9.

\section{References}

1. Goldenblat I.I., Kopnov V.A. Strength criterion for anisotropic materials. Izv. AN SSSR. Mekhanika, 1965, no. 6, pp. 77-83. (In Russian).

2. Bazhanov V.L., Goldenblat I.I., Kopnov V.A., Pospelov A.D., Sinyukov A.M. Plastinki i Obolochki iz Stekloplastikov [Fiberglass Plates and Shells]. M, Vysshaya Shkola Publ., 1970, 408 p. (In Russian).

3. Kopnov V.A., Belov G.P. Evaluating the strength of composite materials and other media with different types of anisotropy. Izvestiya RAN. MTT, 2014, no. 2 (32), pp. 73-80. (In Russian).

4. Giginjak F.F., Kovalchuk B.I., Lamashevsky V.P., Lebedev A.A. Handbook of Mechanical Properties of Structural Materials at a Complex Stress State. Begell House Inc. Publ., 2001, 504 p.

5. Pisarenko G.S., Lebedev A.A. Deformation and strength of materials at a complex stress state. Prikladnaya Mekhanika, 1968, no. 4, iss. 3, pp. 45-50. (In Russian).

6. Fisher L. How to predict structural behavior of R.P. Laminates. Modern Plastics, 1960, no. 6 7. Zakharov K.V. Strength criterion for layered masses. Plasticheskie Massy, 1961, no. 8. (In Russian).

8. Malmeyster A.K. Geometry of strength theories. Mekhanika Polimerov, 1966, no. 4. (In Russian).

9. $\quad$ Alikin V.N., Litvin I.E., Sesyunin S.G., Sokolovsky M.I., Ushin N.V. Kriterii Prochnosti i Nadezhnost Konstruktsiy, pod red. chl.-kor. RAN M.I. Sokolovskogo [Criteria for the Strength and Reliability of Structures, M.I. Sokolovskiy, cor. memb. RAS, ed.]. Moscow, Nedra-Biznestsentr Publ., 2005, 164 p. (In Russian). 
10. Aliev M.M., Shafieva S.V., Karimova N.G. Criteria for the strength and fracture of various materials with due regard for the effect of comprehensive pressure. Vestnik CHGPU im. I.Ya. Yakovleva. Ser. Mekhanika predelnogo sostoyaniya, 2012, no. 3 (13), pp. 64-71. (In Russian).

11. Aliev M.M., Bayburova M.M. Anisotropic materials short-time strength criteria and their application to limit state problems. Vestnik SamGU - Estestvennonauchnaya seriya, 2007, no. 6 (56), pp. 22-29. (In Russian).

12. Bendyukov V.V., Osyayev O.G. Strength criteria for anisotropic composite materials. Nauchnyy vestnik MGTU GA, 2011, no. 163, pp. 151-156. (In Russian).

13. Makovenko S.Ya. The Comparative Analysis of two Criteria of Anisotropic Material Strength. Stroitelnaya Mekhanika Inzhenernykh Konstruktsiy i Sooruzheniy, 2005, no. 1, pp. 65-70. (In Russian).

14. Nekliudova E.A., Semenov A.S., Melnikov B.E., Semenov S.G. Experimental research and finite element analysis of elastic and strength properties of fiberglass composite material. Magazine of Civil Engineering, 2014, no. 3, pp. 25-39. DOI: 10.5862/MCE.47.3

15. Polilov A.N., Tatus N.A. Experimental substantiation of strength criteria for FRP showing directional type of fracture. Vestnik PNIPU, Mekhanika, 2012, no. 2, pp. 140-166. (In Russian).

16. Grebenyuk S.N., Melashchenko O.P. The use of various criteria for calculating the strength of fibrous composites. Zbirnyk Naukovykh Prats Kharkivskogo Universitetu Povitryanykh Sil, 2012, no. 3(32), pp. 134-136. (In Russian).

17. Galicki J., Czech M. A new approach to formulate the general strength theories for anisotropic discontinuous materials. Part A: The experimental base for a new approach to formulate the general strength theories for anisotropic materials on the basis of wood. Applied Mathematical Modelling, 2013, vol. 37, no. 3, pp. 815-827. DOI: 10.1016/j.apm.2012.03.004

18. Niu J., Liu G., Tian J., Zhang Y., Meng L. Comparison of yield strength theories with experimental results. Engineering Mechanics, 2014, vol. 31, no. 1, pp. 181-187. DOI: 10.6052/j.issn.10004750.2012.09.0622

19. Liu G. A novel limiting strain energy strength theory. Transactions of Nonferrous Metals Society of China, 2009, vol. 19, no. 6, pp. 1651-1662. DOI: 10.1016/S1003-6326(09)60084-4

20. Zhang S., Song B., Wang X., Zhao D., Chen X. Deduction of geometrical approximation yield criterion and its application. Journal of Mechanical Science and Technology, 2014, vol. 28, no. 6, pp. 2263-2271. DOI: 10.1007/s12206-014-0515-6

21. Zhu X.-K., Leis B.N. Average shear stress yield criterion and its application to plastic collapse analysis of pipelines. International Journal of Pressure Vessels and Piping, 2006, vol. 83, no. 9, pp. 663-671. DOI: 10.1016/j.ijpvp.2006.06.001

22. Kalnins A., Updike D.P. Limit Pressures of Cylindrical and Spherical Shells. Journal of Pressure Vessel Technology, 2001, vol. 123, no. 3, pp. 288-292. DOI: 10.1115/1.1367273

23. Zezin Y.P. Experimental investigation of the strength properties of particulate polymeric composites, 2016, vol. 1785, pp. 030036. DOI: 10.1063/1.4967057

24. Yan L., Junhai Z., Ergang X., Xueye C. Research on burst pressure for thin-walled elbow and spherical shell made of strength differential materials. Materials Research Innovations, 2015, vol. 19, no. 5, pp. 80-87. DOI: 10.1179/1432891715Z.0000000001340

25. Shroff S., Kassapoglou C. Progressive failure modelling of impacted composite panels under compression. Journal of Reinforced Plastics and Composites, 2015, vol. 34, no. 19, pp. 1603-1614. DOI: $10.1177 / 0731684415592485$

26. Sengupta J., Ghosh A., Chakravorty D. Progressive Failure Analysis of Laminated Composite Cylindrical Shell Roofs. Journal of Failure Analysis and Prevention, 2015, vol. 15, no. 3, pp. 390-400. DOI: 10.1007/s11668-015-9951-6

27. Shokrieh M.M., Karamnejad A. Investigation of Strain Rate Effects on the Dynamic Response of a Glass/Epoxy Composite Plate under Blast Loading by Using the Finite-Difference 
Method. Mechanics of Composite Materials, 2014, vol. 50, no. 3, pp. 295-310. DOI: 10.1007/s11029-014-9415-1

28. Günel M., Kayran A. Non-linear progressive failure analysis of open-hole composite laminates under combined loading. Journal of Sandwich Structures \& Materials, 2013, vol. 15, no. 3, pp. 309-339. DOI: 10.1177/1099636213483651

29. Van der Meer F.P., Sluys L.J., Hallett S.R., Wisnom M.R. Computational modeling of complex failure mechanisms in laminates. Journal of Composite Materials, 2012, vol. 46, no. 5, pp. 603-623. DOI: 10.1177/0021998311410473

30. Pietropaoli E. Progressive Failure Analysis of Composite Structures Using a Constitutive Material Model (USERMAT) Developed and Implemented in ANSYS C. Applied Composite Materials, 2012, vol. 19, no. 3-4, pp. 657-668. DOI: 10.1007/s10443-011-9220-0

31. Garnich M.R., Akula V.M. Review of Degradation Models for Progressive Failure Analysis of Fiber Reinforced Polymer Composites. Applied Mechanics Reviews, 2009, vol. 62, no. 1, pp. 010801. DOI: 10.1115/1.3013822

32. Bleyer J., de Buhan P. A numerical approach to the yield strength of shell structures. European Journal of Mechanics - A/Solids, 2016, vol. 59, pp. 178-194. DOI: 10.1016/j.euromechsol.2016.03.002

33. Sun H.-H., Tan P.-L. Background of ABS Buckling Strength Assessment Criteria for Cylindrical Shells in Offshore Structures. Journal of Offshore Mechanics and Arctic Engineering, 2008, vol. 130, no. 2, pp. 021012. DOI: 10.1115/1.2913349

34. Mellor P.B. The ultimate strength of thin-walled shells and circular diaphragms subjected to hydrostatic pressure. International Journal of Mechanical Sciences, 1960, vol. 1, nos. 2-3, pp. 216228. DOI: 10.1016/0020-7403(60)90041-2

35. Noh H.C. Ultimate strength of large scale reinforced concrete thin shell structures. Thin-Walled Structures, 2005, vol. 43, no. 9, pp. 1418-1443. DOI: 10.1016/j.tws.2005.04.004

36. Zhang B., Sun Q. The Ultimate Strength of Stiffened Panel with Overall Buckling. Advanced Materials Research, 2011, vol. 308-310, pp. 1297-1301. DOI: 10.4028/www.scientific.net/AMR.308310.1297

37. Ueda Y., Rashed S.M.H., Paik J.K. Buckling and ultimate strength interaction in plates and stiffened panels under combined inplane biaxial and shearing forces. Marine Structures, 1995, vol. 8, no. 1, pp. 1-36. DOI: 10.1016/0951-8339(95)90663-F

38. Abrosimov N.A., Elesin A.V. Numerical analysis of dynamic strength of composite cylindrical shells under multiple-pulse exposures. PNRPU Mechanics Bulletin, 2016, no. 4, pp. 7-19. DOI: $10.15593 /$ perm.mech/2016.4.01

39. Karpov V.V., Semenov A.A. Strength criteria for thin orthotropic shells. Part 2: Calculation and analysis. Vestnik Grazhdanskikh Inzhenerov, 2015, no. 1 (48), pp. 60-70. (In Russian).

40. Tsvetkov S.V., Kulish G.G. Strength Criteria of Unidirectional Organic Plastic in Three-Axis Stress State. Vestnik MGTU im. N.E. Baumana. Seriya: Mashinostroe, 2011, no. SP, pp. 19-28. (In Russian).

41. $\mathrm{Yu} \mathrm{M.-H.} \mathrm{Advances} \mathrm{in} \mathrm{strength} \mathrm{theories} \mathrm{for} \mathrm{materials} \mathrm{under} \mathrm{complex} \mathrm{stress} \mathrm{state} \mathrm{the} \mathrm{20th}$ sentury. Appl. Mech. Rev., 2002, vol. 55, no. 3, pp. 169-218. DOI: 10.1115/1.1472455

42. Yu M.-H., Li J.-C. Computational plasticity: with emphasis on the application of the unified strength theory. Hangzhou, Zhejiang Univ. Press, 2012, 529 p.

43. Kolupaev V.A., Yu M.-H., Altenbach H. Visualization of the Unified Strength Theory. Archive of Applied Mechanics, 2013, vol. 83, no. 7, pp. 1061-1085. DOI: 10.1007/s00419-013-0735-8

44. Tsvetkov S.V. Strength criteria for transversely isotropic materials of different symmetry classes of structure. Vestnik MGTU im. N.E. Baumana. Seriya: Mashinostroenie, 2009, no. 1, pp. 86-99. (In Russian).

45. Karpov V.V., Semenov A.A. Strength criteria for thin orthotropic shells. Part 1: Analysis of the basic strength criteria for isotropic and orthotropic materials. Vestnik Grazhdanskikh Inzhenerov, 2014, no. 6 (47), pp. 43-51. (In Russian). 
46. Smerdov A.A., Buyanov I.A., Chudnov I.V. Analysis of optimal combinations of requirements to developed CFRP for large space-rocket designs. Izvestiya Vuzov. Mashinostroenie, 2012, no. 8, pp. 70-77. (In Russian).

47. Tyshkevich V.N. The choice of strength criteria for pipes made of reinforced plastics. Izvestiya Volgogradskogo Gosudarstvennogo Tekhnicheskogo Universiteta, 2011, no. 5 (78), pp. 76-79. (In Russian).

48. Karpov V.V., Semenov A.A. Mathematical models and algorithms for studying strength and stability of shell structures. Journal of Applied and Industrial Mathematics, 2017, vol. 11, no. 1, pp. 70-81. DOI: 10.1134/S1990478917010082

49. Kuznetsov E.B. Continuation of solutions in multiparameter approximation of curves and surfaces. Computational Mathematics and Mathematical Physics, 2012, vol. 52, no. 8, pp. 1149-1162. DOI: $10.1134 / \mathrm{S} 0965542512080076$ 
Подана в журнал: 09.01.2018

УДК 539.3, 539.4

DOI: $10.17804 / 2410-9908.2018 .1 .016-033$

\title{
АНАЛИЗ ПРОЧНОСТИ ОБОЛОЧЕЧНЫХ КОНСТРУКЦИЙ ИЗ СОВРЕМЕННЫХ МАТЕРИАЛОВ В СООТВЕТСТВИИ С РАЗЛИЧНЫМИ КРИТЕРИЯМИ ПРОЧНОСТИ
}

\author{
А. А. Семенов \\ Санкт-Петербургский государственный архитектурно-строительный университет, \\ 190005, 2-я Красноармейская ул., 4, Санкт-Петербург, Российская Федерация \\ 目 sw.semenov@gmail.com; $\mathbb{D}$ https://orcid.org/0000-0001-9490-7364 \\ Ответственный автор. Электронная почта: sw.semenov@gmail.com \\ Адрес для переписки: 190005, г. Санкт-Петербург, ул. 2-я Красноармейская, д. 4, Российская Федерация
}

В работе проанализирована возможность применения пяти различных критериев прочности (критерий максимальных напряжений, Мизеса - Хилла, Писаренко - Лебедева, Фишера, Гольденблата - Копнова) к расчетам прочности ортотропных оболочечных конструкций.

Рассматрены пологие оболочки двоякой кривизны, квадратные в плане, панели цилиндрических и конических оболочек. Использована геометрически нелинейная математическая модель их деформирования, учитывающая поперечные сдвиги. Для расчетов использованы характеристики современных ортотропных материалов - стеклопластика и углепластика. Показано развитие областей невыполнения условий прочности при увеличении нагрузки.

Ключевые слова: прочность, критерий прочности, теория прочности, оболочки, углепластик.

\section{1. Введение}

Прочность конструкций, подверженных воздействию внешних нагрузок, анализируется по предельному состоянию материалов, из которых эти конструкции изготовлены.

Для изотропных материалов основные критерии прочности были разработаны в конце 19-го века (Г. Галилей, Г. Ламе, А. Клебша, Ф. Мариотт, М. Навье, Ш. О. Кулон, И. Баушингер, И. Максвелл, М. Т. Губер, Р. Мизес, Н. Генки и др.).

Критерии прочности анизотропных материалов можно найти в работах И. И. Гольденблата [1-2], В. А. Копнова [1-3], Б. И. Ковальчука [4], А. А. Лебедева [4-5], Г. С. Писаренко [5], Л. Фишера [6], Р. Хилла и др.

Из анализа критериев прочности, применимых для ортотропных материалов [1-37], следует, что не существует одного универсального критерия прочности, поэтому приходится использовать несколько критериев с последующим анализом полученных результатов. Результаты таких сравнений можно найти в работах $[16,18,26,38,39]$.

В исследованиях, посвященных прочности материалов и конструкций, достаточно часто встречаются критерий максимальных напряжений [26, 38], критерий Гольденблата Копнова $[12,13]$, критерий Цая-Ву [26, 28, 31, 40], критерий Хоффмана $[17,26,38]$, критерий Хашина [25, 27, 28, 30].

Среди недавних работ по развитию и применению теорий прочности следует отметить работы Mao-Hong Yu [41-43], С. В. Цветкова [40, 44], Н. А. Абросимова и А. В. Елесина [38].

Сравнительно мало работ посвящено применению различных теорий прочности к расчету оболочечных конструкций $[22,24,26,32-35,38]$. 


\section{2. Постановка задачи и методы решения}

Цель данной работы - проведение анализа прочности различных по геометрии оболочечных конструкций, выполненных из современных ортотропных материалов, на основе различных критериев прочности.

Рассматрим пять критериев для случая плоского напряженного состояния, которые в единых обозначениях могут быть представлены следующим образом $[39,45]$ :

- Критерий 1 - критерий максимальных напряжений

$$
F_{1}^{-} \leq \sigma_{11} \leq F_{1}^{+}, \quad F_{2}^{-} \leq \sigma_{22} \leq F_{2}^{+}, \quad\left|\tau_{12}\right| \leq F_{12}
$$

- Критерий 2 - критерий Мизеса - Хилла

$$
\frac{\sigma_{11}^{2}}{F_{1}^{2}}-\frac{\sigma_{11} \sigma_{22}}{F_{1} F_{2}}+\frac{\sigma_{22}^{2}}{F_{2}^{2}}+\frac{\tau_{12}^{2}}{F_{12}^{2}} \leq 1
$$

- Критерий 3 - критерий Писаренко - Лебедева

$$
\sqrt{(A+C) \sigma_{11}^{2}-2 A \sigma_{11} \sigma_{22}+(A+B) \sigma_{22}^{2}}+D_{1} \sigma_{11}+D_{2} \sigma_{22} \leq 1
$$

Здесь

$$
\begin{aligned}
& A=\frac{1}{2}\left(K^{2}-\frac{1}{\left(F_{1}^{-}\right)^{2}}-\frac{1}{\left(F_{2}^{-}\right)^{2}}\right), \quad B=\frac{1}{2}\left(\frac{1}{\left(F_{1}^{-}\right)^{2}}+\frac{3}{\left(F_{2}^{-}\right)^{2}}-K^{2}\right), \\
& C=\frac{1}{2}\left(\frac{1}{\left(F_{2}^{-}\right)^{2}}+\frac{3}{\left(F_{1}^{-}\right)^{2}}-K^{2}\right), \quad D_{1}=\frac{1}{F_{1}^{+}}+\frac{1}{F_{1}^{-}}, \quad D_{2}=\frac{1}{F_{2}^{+}}+\frac{1}{F_{2}^{-}}, \quad K=-\frac{1}{F_{12,45}^{-}}-\frac{1}{F_{2}^{+}}-\frac{1}{F_{2}^{-}} .
\end{aligned}
$$

- Критерий 4 - критерий Фишера

$$
\frac{\sigma_{11}^{2}}{F_{1}^{2}}-K_{f} \frac{\sigma_{11} \sigma_{22}}{F_{1} F_{2}}+\frac{\sigma_{22}^{2}}{F_{2}^{2}}+\frac{\tau_{12}^{2}}{F_{12}^{2}} \leq 1, \quad \text { где } K_{f}=\frac{E_{1}\left(1+\mu_{21}\right)+E_{2}\left(1+\mu_{12}\right)}{2 \sqrt{E_{1} E_{2}\left(1+\mu_{21}\right)\left(1+\mu_{12}\right)}} .
$$

- Критерий 5 - критерий Гольденблата - Копнова

$$
\begin{aligned}
& \frac{1}{2}\left(\frac{1}{F_{1}^{+}}+\frac{1}{F_{1}^{-}}\right) \sigma_{11}+\frac{1}{2}\left(\frac{1}{F_{2}^{+}}+\frac{1}{F_{2}^{-}}\right) \sigma_{22}+\frac{1}{2}\left\{\left(\frac{F_{1}^{+}-F_{1}^{-}}{F_{1}^{+} F_{1}^{-}}\right)^{2} \sigma_{11}^{2}+\left(\frac{F_{2}^{+}-F_{2}^{-}}{F_{2}^{+} F_{2}^{-}}\right)^{2} \sigma_{22}^{2}+\right. \\
& \left.+\left[\left(\frac{F_{1}^{+}-F_{1}^{-}}{F_{1}^{+} F_{1}^{-}}\right)^{2}+\left(\frac{F_{2}^{+}-F_{2}^{-}}{F_{2}^{+} F_{2}^{-}}\right)^{2}-\left(\frac{F_{12,45}^{+}-F_{12,45}^{-}}{F_{12,45}^{+} F_{12,45}^{-}}\right)^{2}\right] \sigma_{11} \sigma_{22}+\frac{4 \tau_{12}^{2}}{F_{12}^{2}}\right\}^{\frac{1}{2}} \leq 1 .
\end{aligned}
$$


Для критериев 2 и 4 имеет место условие:

$$
F_{1}=\left\{\begin{array}{l}
F_{1}^{+} \text {при } \sigma_{11} \geq 0, \\
F_{1}^{-} \text {при } \sigma_{11}<0,
\end{array} \quad F_{2}=\left\{\begin{array}{l}
F_{2}^{+} \text {при } \sigma_{22} \geq 0, \\
F_{2}^{-} \text {при } \sigma_{22}<0 .
\end{array}\right.\right.
$$

Необходимо учитывать, что перед использованием критериев Писаренко - Лебедева и Гольденблата - Копнова необходимо проверить условие совместности прочностных характеристик материала (с учетом принятых обозначений):

$$
\left(\frac{1}{F_{1}^{+}}+\frac{1}{F_{1}^{-}}\right)-\left(\frac{1}{F_{2}^{+}}+\frac{1}{F_{2}^{-}}\right)-\left(\frac{1}{F_{12,45}^{+}}+\frac{1}{F_{12,45}^{-}}\right)=0 .
$$

Следует отметить, что в некоторых источниках в предельных значениях сжимающих напряжений знак «-» не показан. Для единообразия и согласованности записи критериев с вычисляемыми значениями предполагается, что значения сжимающих напряжений, а также их предельные значения, имеют знак «-».

В формулировках критериев приняты следующие обозначения:

1, 2, 3 - ортогональная система координат, соответствующая осям ортотропии материала;

$E_{1}, E_{2}$ - модули упругости в направлениях ортотропии 1,2 [МПа];

$\mu_{12}, \mu_{21}-$ коэффициенты Пуассона;

$F_{1}^{+}, F_{2}^{+}$- предел прочности при растяжении в направлениях ортотропии 1,2 [МПа];

$F_{1}^{-}, F_{2}^{-}-$предел прочности при сжатии в направлениях ортотропии 1,2 [МПа];

$F_{12}$ - предел прочности при сдвиге в плоскости ортотропии [МПа];

$F_{12,45}^{+}, F_{12,45}^{-}$- пределы прочности на сдвиг по площадкам, наклоненным к основным под углом $45^{\circ}[\mathrm{MПа}]$;

$\sigma_{11}, \sigma_{22}, \sigma_{33}$ - нормальные напряжения в направлении осей ортотропии 1, 2, 3 [МПа];

$\tau_{12}, \tau_{13}, \tau_{23}$ - касательные напряжения в плоскостях 1O2, 103, $2 \mathrm{O} 3$ [МПа].

Если оси ортотропии материала 1, 2 не совпадают с осями координат конструкции $x, y$, то напряжения в конструкции $\sigma_{x}, \sigma_{y}, \tau_{x y}$ необходимо привести к направлениям осей ортотропии 1,2 , используя формулы поворота системы координат. В таком случае в формулах критериев прочности можно будет использовать известные значения пределов прочности материала.

В дальнейшем будем считать, что оси ортотропии 1,2 совпадают с осями принятой локальной системы координат $x, y$ соответственно $(x, y, z$ - ортогональная система координат в срединной поверхности оболочной конструкции; $x, y$ - криволинейные координаты, направленные по линиям главных кривизн, $z$ - координата, направленная в сторону вогнутости оболочки, перпендикулярно срединной поверхности).

\section{3. Результаты и обсуждение}

Механические характеристики материалов рассматриваемых конструкций представлены в табл. 1.

Будем рассматривать пологие ортотропные оболочечные конструкции двоякой кривизны, прямоугольные в плане (рис. 1 a), а также цилиндрические (рис. 1 б) и конические панели, шарнирно-неподвижно закрепленные по контуру и находящиеся под действием 
внешней равномерно распределенной поперечной нагрузки $q$. Нагрузка направлена по нормали к срединной поверхности.

Таблица 1 - Механические характеристики материалов рассматриваемых конструкций

\begin{tabular}{|c|c|c|c|}
\hline \multirow{2}{*}{ Характеристика } & \multicolumn{2}{|c|}{ Углепластик [46] } & \multirow{2}{*}{$\frac{\text { Стеклопластик [47] }}{\text { T-10/УПЭ22-27 }}$} \\
\hline & ЛУ-П/ЭНФБ & M60J/Eроху & \\
\hline$E_{1}, \mathrm{M \Pi la}$ & $1,4 \cdot 10^{5}$ & $3,3 \cdot 10^{5}$ & $0,294 \cdot 10^{5}$ \\
\hline$\mu_{12}$ & 0,3 & 0,32 & 0,123 \\
\hline$E_{2}, \mathrm{M \Pi la}$ & $0,97 \cdot 10^{4}$ & $0,59 \cdot 10^{4}$ & $1,78 \cdot 10^{4}$ \\
\hline$G_{12}, \mathrm{MПа}$ & $0,46 \cdot 10^{4}$ & $0,39 \cdot 10^{4}$ & $0,301 \cdot 10^{4}$ \\
\hline$G_{13}, \mathrm{M \Pi la}$ & $0,46 \cdot 10^{4}$ & $0,39 \cdot 10^{4}$ & $0,301 \cdot 10^{4}$ \\
\hline$G_{23}, \mathrm{MПla}$ & $0,46 \cdot 10^{4}$ & $0,39 \cdot 10^{4}$ & $0,301 \cdot 10^{4}$ \\
\hline$F_{1}^{+}, \mathrm{M \Pi а}$ & 700 & 1760 & 508 \\
\hline$F_{1}^{-}, \mathrm{MПа}$ & -600 & -780 & -209 \\
\hline$F_{2}^{+}, \mathrm{M \Pi la}$ & 27 & 30 & 246 \\
\hline$F_{2}^{-}, \mathrm{M \Pi \textrm {a }}$ & -184 & -168 & -117 \\
\hline$F_{12}, \mathrm{MПа}$ & 55 & 39 & 43 \\
\hline$F_{12,45}^{+}, \mathrm{MПla}$ & - & - & 130 \\
\hline$F_{12,45}^{-}, \mathrm{MПla}$ & - & - & -160 \\
\hline
\end{tabular}

Здесь $G_{12}, G_{13}, G_{23}$ - модули сдвига в плоскостях ортотропии.

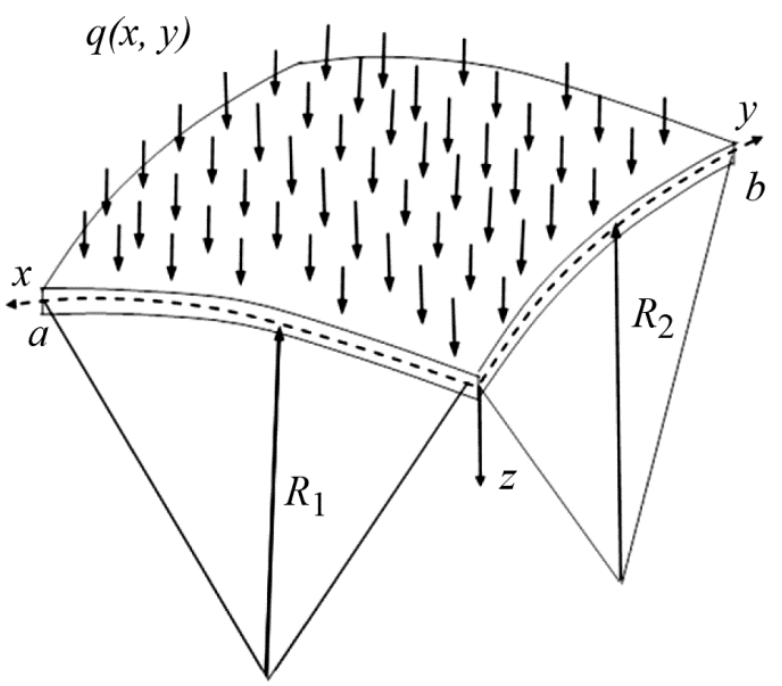

$a$

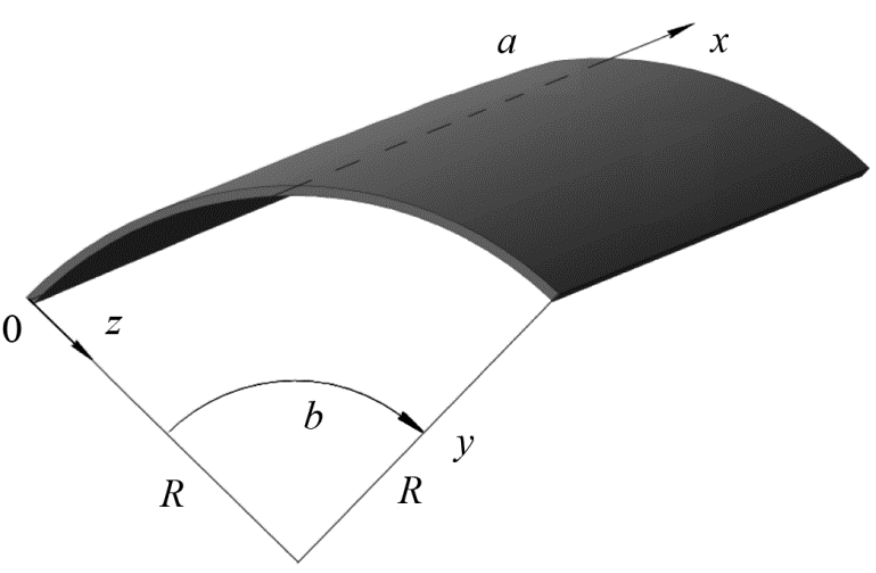

$\sigma$

Рис. 1. Схематичное изображение пологой оболочки, прямоугольной в плане и цилиндрической панели 
Математическая модель деформирования таких конструкций строится из трех групп соотношений: геометрических (связывающих перемещения и деформации), физических (связывающих напряжения и деформации) и функционала полной потенциальной энергии деформации.

Рассматрим геометрически нелинейный вариант модели, также учитывающий ортотропию материала и поперечные сдвиги (модель типа Тимошенко). В таком случае, неизвестными функциями будут три функции перемещений $U=U(x, y) ; V=V(x, y) ; W=W(x, y)$ и две функции углов поворота нормали $\Psi_{x}=\Psi_{x}(x, y) ; \Psi_{y}=\Psi_{y}(x, y)$. Подробно используемая математическая модель была рассмотрена в работе [48].

К модели был применен метод Ритца для сведения задачи нахождения минимума функционала к решению системы нелинейных алгебраических уравнений. Полученную систему решали методом продолжения решения по наилучшему параметру [49]. Программная реализация осуществлена в среде аналитических вычислений Maple 2016.

Анализ прочности материала конструкций проведем на примере следующих вариантов оболочек.

1. Пологая оболочка, квадратная в плане, с линейными размерами $a=b=600 h$, главными радиусами кривизны $R_{1}=R_{2}=1510 h$ и толщиной $h=0,09$ м, выполненная из углепластика M60J/Eроху.

2. Панель цилиндрической оболочки с параметрами $a=20 \mathrm{M} ; R=5,4 \mathrm{M} ; h=0,01 \mathrm{м}$ и углом разворота $\pi$ рад, выполненная из углепластика ЛУ-П/ЭНФБ.

3. Панель конической оболочки с параметрами $a_{1}=50 \mathrm{м} ; a=90 \mathrm{м} ; \theta=0,78$ рад; $h=0,01$ м, углом разворота $\pi$ рад, выполненная из стеклопластика Т-10/УПЭ22-27.

Расчеты проводились при удержании 16 членов в разложении искомых функций в ряды в методе Ритца $(N=16)$. Для исследования прочности указанных оболочечных конструкций будем рассматривать следующие критерии:

- критерий максимальных напряжений (на графиках кривая Max, черный цвет);

- критерий Мизеса - Хилла (кривая $M-H$, зеленый цвет);

- критерий Фишера (кривая $F$, розовый цвет);

- критерий Гольденблата - Копнова (кривая $G-K$, коричневый цвет);

- критерий Писаренко - Лебедева (кривая $P-L$, фиолетовый цвет).

Следует отметить, что из рассматриваемых вариантов оболочек критерии Гольденблата - Копнова и Писаренко - Лебедева применимы только к варианту 3, так как для остальных рассматриваемых материалов не выполняется условие совместности прочностных характеристик из-за отсутствия данных о предельных значениях $\tau_{12,45}^{+}, \tau_{12,45}^{-}$.

На рис. 2-4 представлены графики зависимости значений выбранных критериев $K_{r}$ от нагрузки $q$. Здесь $K_{r}$ - левая часть критерия прочности, для критерия максимальных напряжений левую часть будем брать в виде $K_{r}=\max \left\{\left|\frac{\sigma_{11}}{F_{1}}\right|,\left|\frac{\sigma_{22}}{F_{2}}\right|,\left|\frac{\tau_{12}}{F_{12}}\right|\right\}$. Момент перехода $K_{r}$ через единицу (начало невыполнения условия прочности) на графиках отмечен кружком. В табл. 2 приводятся значения предельных нагрузок начала невыполнения условий прочности, для рассматриваемых вариантов оболочек по выбранным критериям. 


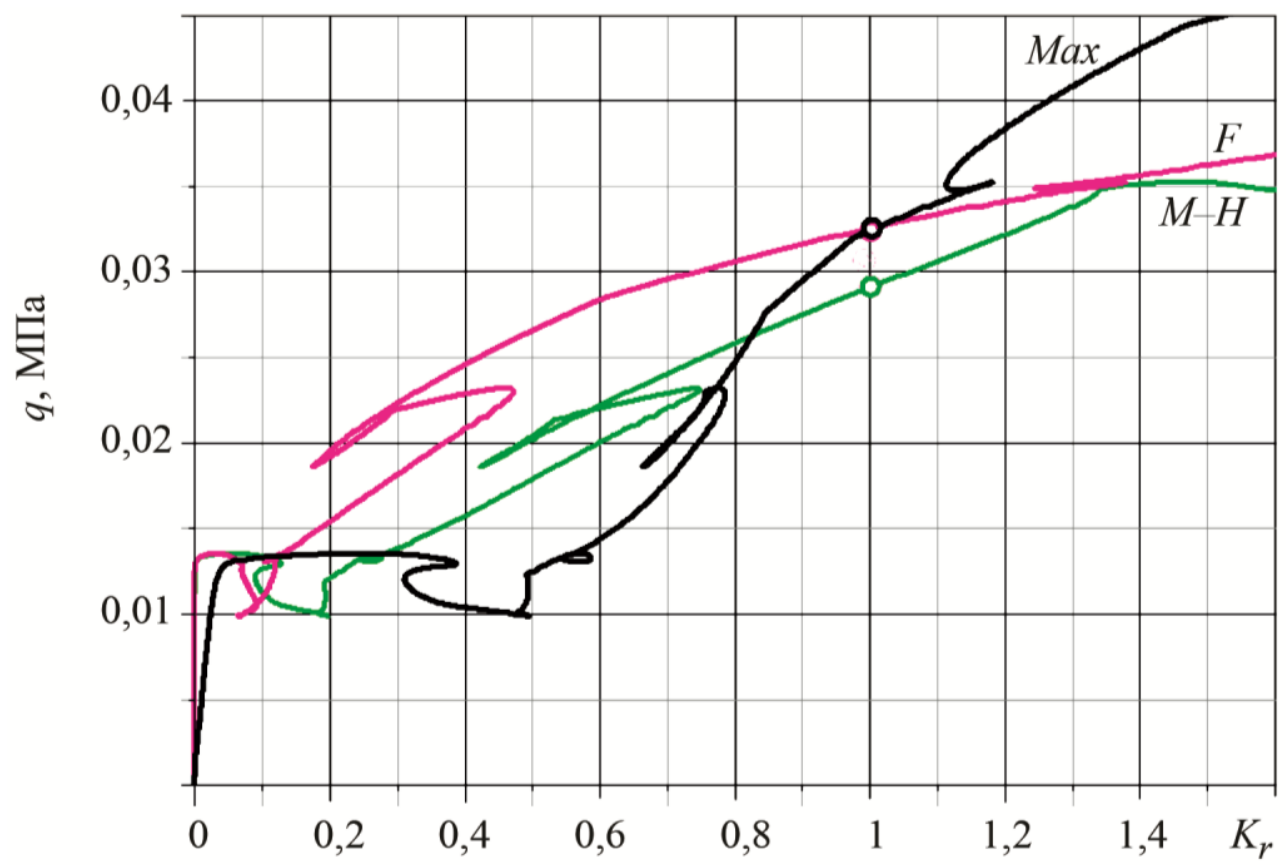

Рис. 2. Кривые зависимости значений выбранных критериев $K_{r}$ от нагрузки $q$ для конструкции варианта 1

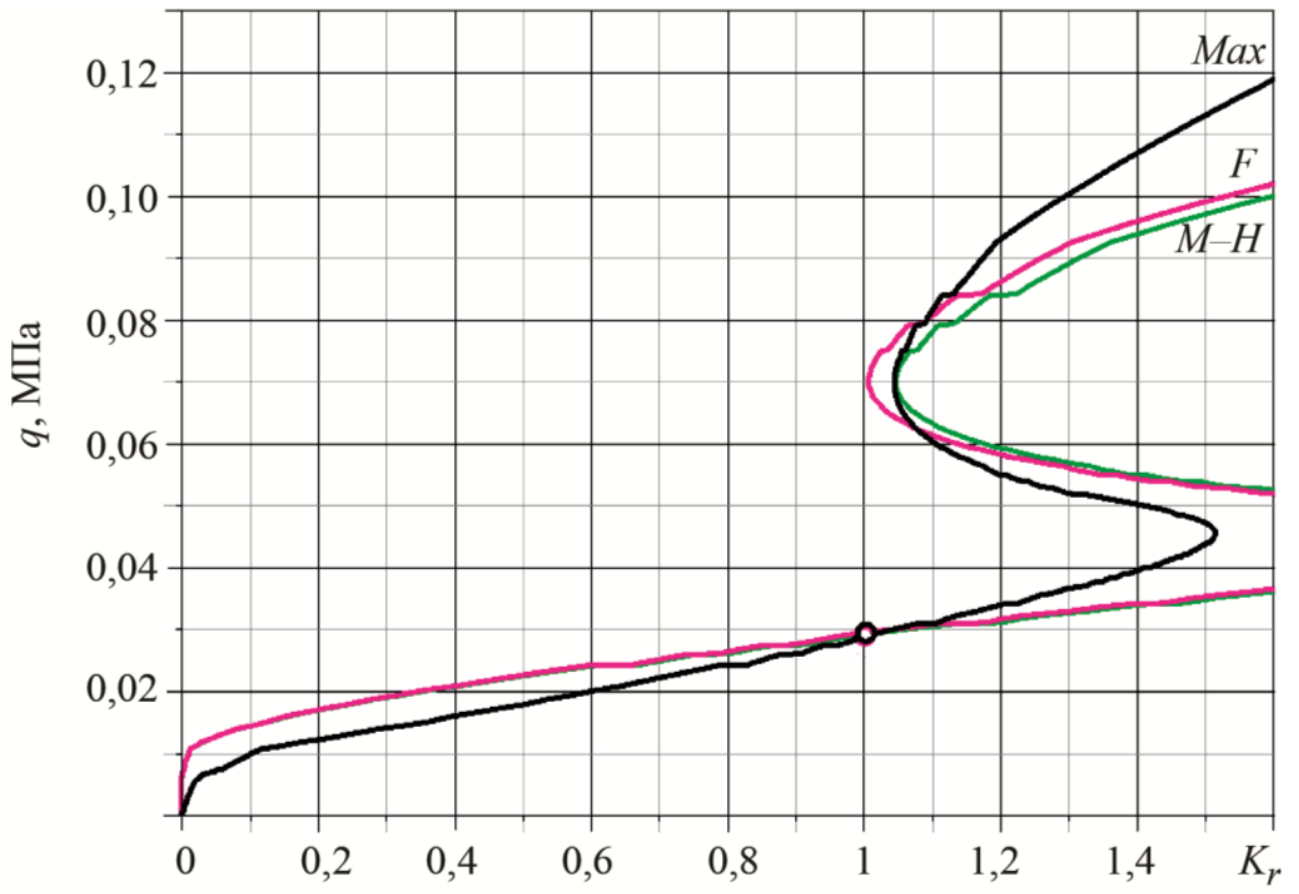

Рис. 3. Кривые зависимости значений выбранных критериев $K_{r}$ от нагрузки $q$ для конструкции варианта 2 


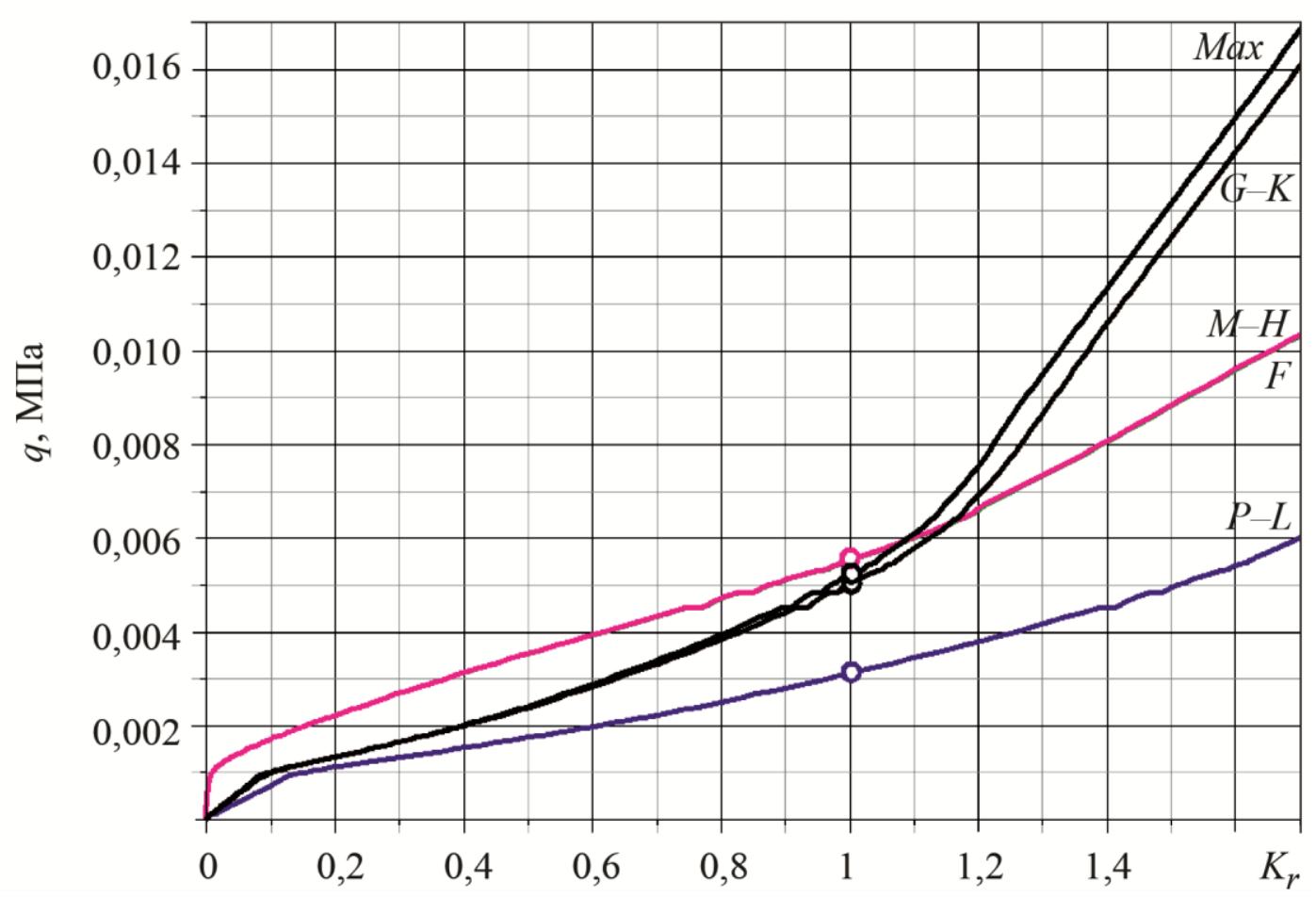

Рис. 4. Кривые зависимости значений выбранных критериев $K_{r}$ от нагрузки $q$ для конструкции варианта 3

Таблица 2 - Значения предельно допустимых нагрузок для рассматриваемых вариантов оболочек в зависимости от критерия прочности

\begin{tabular}{|c|c|c|c|c|c|}
\hline \multirow{2}{*}{ Вариант } & \multicolumn{5}{|c|}{ Предельно допустимая нагрузка $q_{\text {nlin }}$ по критерию } \\
\cline { 2 - 6 } & $\begin{array}{c}\text { Максимальных } \\
\text { напряжений }\end{array}$ & $\begin{array}{c}\text { Мизеса }- \\
\text { Хилла }\end{array}$ & Фишера & $\begin{array}{c}\text { Гольденблата }- \\
\text { Копнова }\end{array}$ & $\begin{array}{c}\text { Писаренко - } \\
\text { Лебедева }\end{array}$ \\
\hline 1 & $0,032, F_{12}$ & 0,029 & 0,033 & - & - \\
\hline 2 & $0,029, F_{2}^{+}$ & 0,030 & 0,030 & - & - \\
\hline 3 & $0,0052, F_{2}^{-}$ & 0,0055 & 0,0055 & 0,0051 & 0,0032 \\
\hline
\end{tabular}

Как видно из рис. 2-4 и табл. 2, различные критерии дают близкие результаты.

В табл. 3 показано развитие областей потери прочности для оболочки варианта 1 при увеличении значения нагрузки уже после начального разрушения материала по критериям максимальных напряжений, Мизеса - Хилла и критерию Фишера. При построении областей для критерия максимальных напряжений учитываются предельные значения по всем трем компонентам напряжений (сделано наложение областей). 
Таблица 3 - Развитие областей потери прочности для оболочки варианта 1

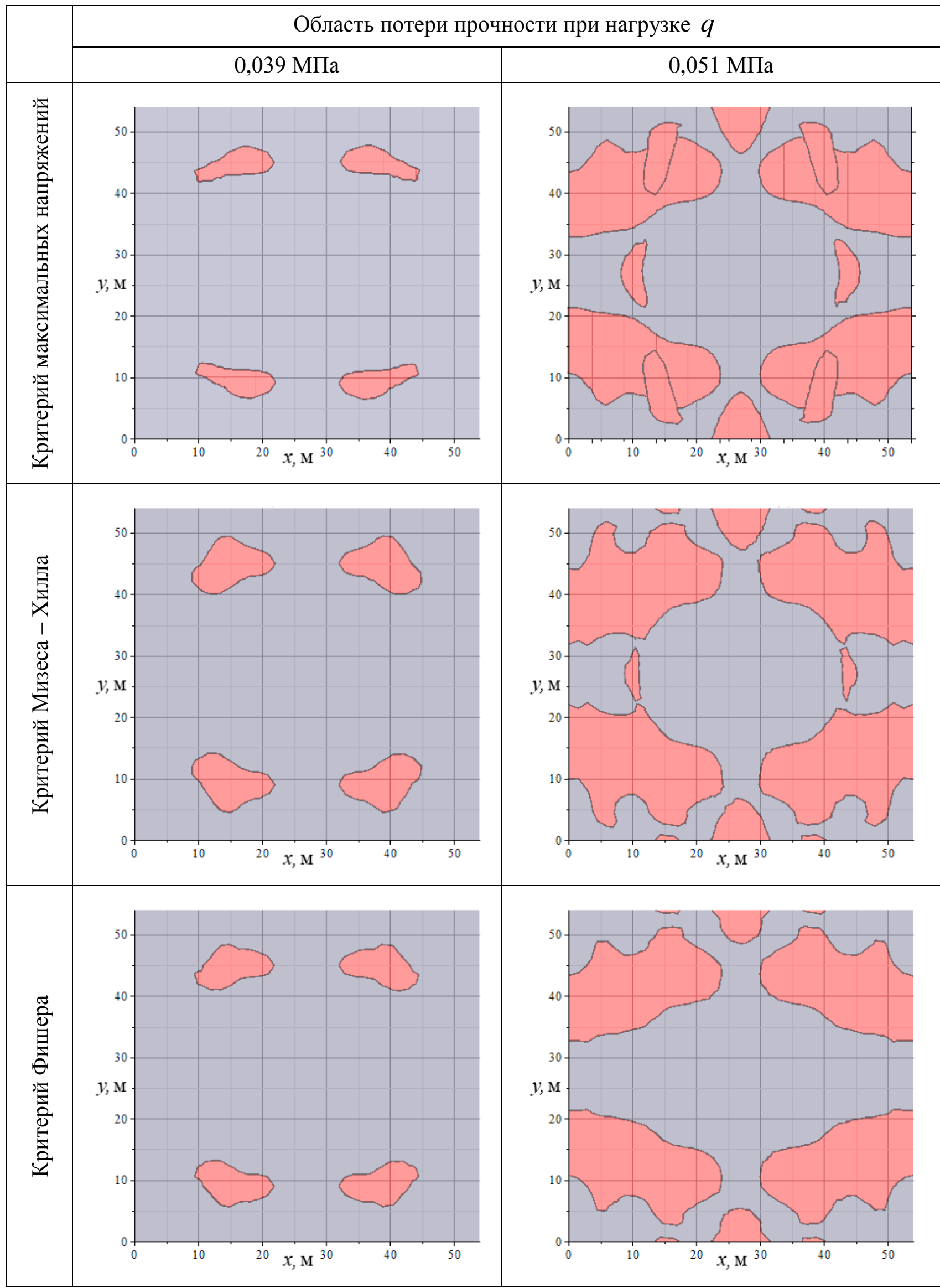

For citation: Semenov A. A. Analysis of the strength of shell structures, made from modern materials, according to various strength criteria // Diagnostics, Resource and Mechanics of materials and structures. - 2018. - Iss. 1. - P. 16-33. DOI: 10.17804/2410-9908.2018.1.016-033. 
Как видно из табл. 3, развитие областей потери прочности для различных критериев имеет схожий характер.

В табл. 4 показано развитие областей потери прочности для оболочки варианта 3 при увеличении значения нагрузки уже после начального разрушения материала по критериям максимальных напряжений, Мизеса - Хилла, Фишера, Гольденблата - Копнова и Писаренко - Лебедева.

Таблица 4 - Развитие областей потери прочности для оболочки варианта 3

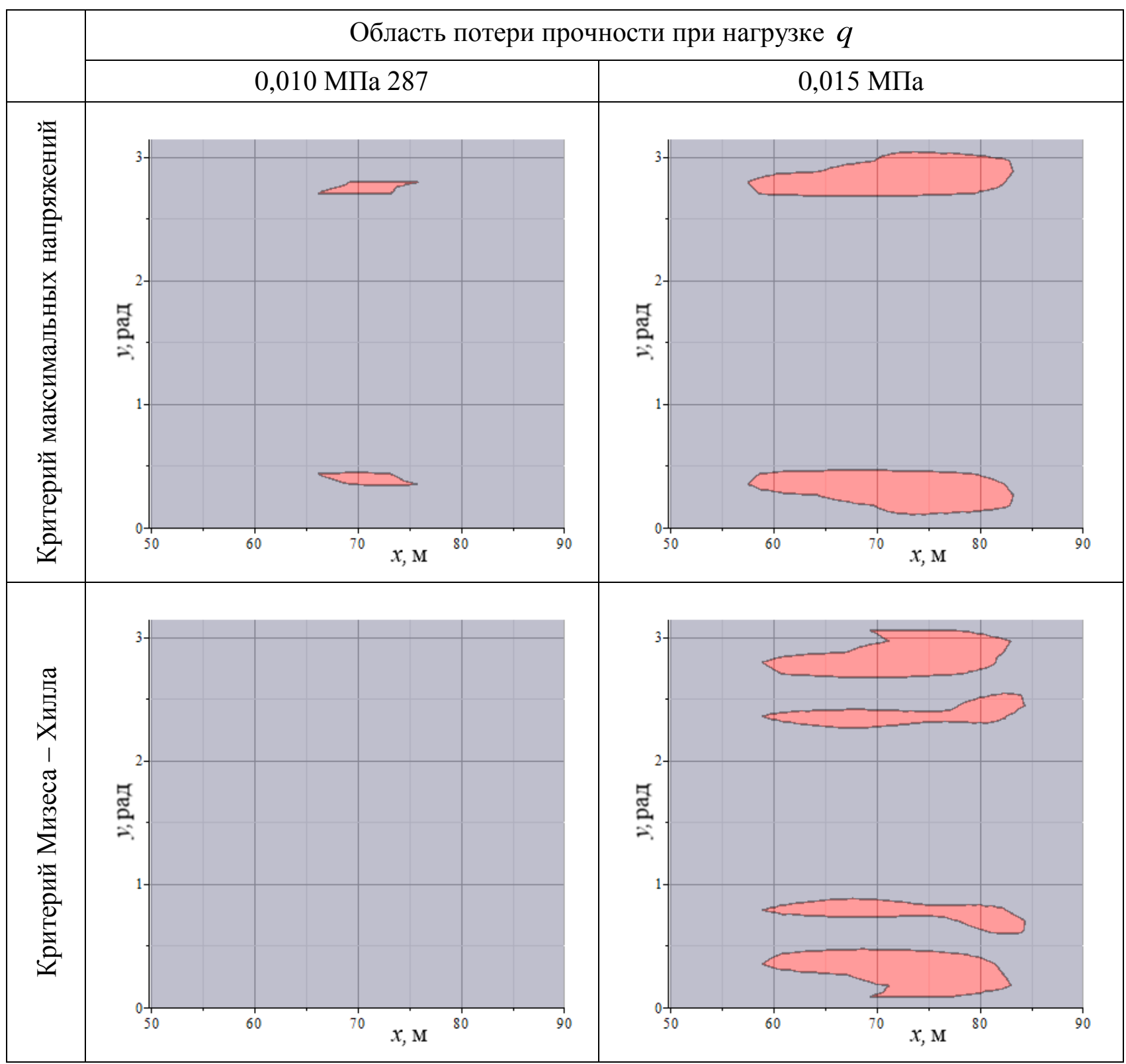




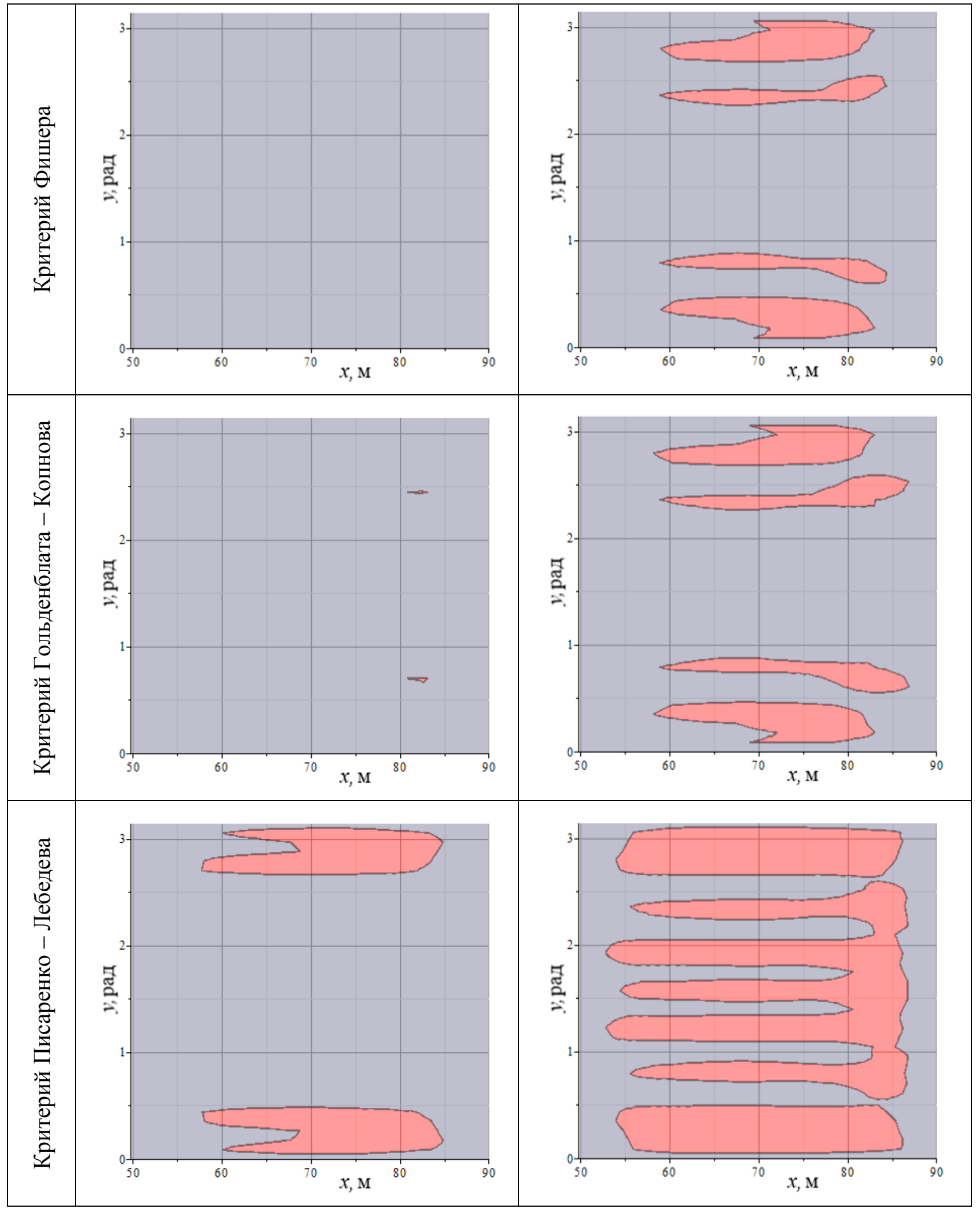

Как видно из рисунков и значений в табл. 2, 3 и 4, критерии максимальных напряжений, Мизеса - Хилла и Фишера дают практически одинаковый результат. Критерий Гольденблата - Копнова дает также близкие к остальным критериям значения, но требует наличия дополнительных данных о материале и выполнения условия совместности. Для критерия 
Писаренко - Лебедева это также необходимо, но он дает результат, сравнительно отличающийся от остальных критериев.

\section{4. Заключение}

Так как предельные нагрузки потери прочности, найденные по критерию максимальных напряжений, практически совпадают с аналогичными нагрузками, найденными по другим критериям, и, кроме того, в отличие от других критериев он еще указывает, по какому компоненту напряжений происходит потеря прочности, то в дальнейших исследованиях прочности оболочечных конструкций будем использовать именно его.

Проведен анализ развития областей невыполнения условий прочности при закритическом деформировании, который показал схожие результаты для разных критериев. Этот анализ дает информацию для оптимальной расстановки ребер жесткости для повышения прочности оболочечных конструкций.

\section{Благодарность}

Работа выполнена при поддержке Минобрнауки РФ в рамках государственного задания, проект № 9.5605.2017/8.9.

\section{Литература}

1. Гольденблат И. И., Копнов В. А. Критерий прочности анизотропных материалов // Изв. АН СССР. Механика. - 1965. - № 6. - С. 77-83.

2. Пластинки и оболочки из стеклопластиков / В. Л. Бажанов, И. И. Гольденблат, В. А. Копнов, А. Д. Поспелов, А. М. Синюков. - М. : Изд-во Высшая школа, 1970. - 408 с.

3. Копнов В. А., Белов Г. П. Оценка прочности композиционных материалов и других сред с различными видами анизотропии // Известия РАН. МТТ. - 2014. - № 2 (32). C. $73-80$.

4. Механические свойства конструкционных материалов при сложном напряженном состоянии : справочник / А. А. Лебедев, Б. И. Ковальчук, Ф. Ф. Гигиняк, В. П. Ламашевский. Киев: Наукова думка, 1983. - 366 с.

5. Писаренко Г. С., Лебедев А. А. Деформирование и прочность материалов при сложном напряженном состоянии // Прикладная механика. - 1968. - № 4, вып. 3. - С. 45-50.

6. Fisher L. How to predict structural behavior of R.P. Laminates // Modern Plastics. - 1960. № 6 .

7. Захаров К. В. Критерий прочности для слоистых масс // Пластические массы. - 1961. № 8 .

8. Малмейстер А. К. Геометрия теорий прочности // Механика полимеров. - 1966. - № 4. 9. Критерии прочности и надежность конструкций / В. Н. Аликин, И. Е. Литвин, С. Г. Сесюнин, М. И. Соколовский, Н. В. Ушин / под ред. чл.-кор. РАН М. И. Соколовского. - М. : ООО «Недра-Бизнесцентр», 2005. - 164 с.

10. Алиев М. М., Шафиева С. В., Каримова Н. Г. Критерии прочности и разрушения разнопрочных материалов с учетом влияния всестороннего давления // Вестник ЧГПУ им. И. Я. Яковлева. Серия: Механика предельного состояния. - 2012. - № 3 (13). - С. 64-71.

11. Алиев М. М., Байбурова М. М. Критерии кратковременной прочности анизотропных материалов и применение их для решения задач предельного равновесия // Вестник СамГУ. Естественнонаучная серия. - 2007. - № 6 (56). - С. 22-29.

12. Бендюков В. В., Осяев О. Г. Критерии прочности анизотропных композитных материалов // Научный вестник МГТУ ГА. - 2011. - № 163. - С. 151-156. 
13. Маковенко С. Я. О взаимности компонент тензоров прочности некоторых теорий прочности анизотропных материалов // Строительная механика инженерных конструкций и сооружений. - 2005. - № 1. - С. 65-70.

14. Experimental research and finite element analysis of elastic and strength properties of fiberglass composite material / E. A. Nekliudova, A. S. Semenov, B. E. Melnikov, S. G. Semenov // Magazine of Civil Engineering. - 2014. - No. 3. - P. 25-39. - DOI: 10.5862/MCE.47.3

15. Полилов А. Н., Татусь Н. А. Экспериментальное обоснование критериев прочности волокнистых композитов, проявляющих направленный характер разрушения // Вестник ПНИПУ. Механика. - 2012. - № 2. - С. 140-166.

16. Гребенюк С. Н., Мелащенко О. П. Использование различных критериев для расчёта прочности волокнистых композитов // Збірник наукових праць Харківського університету Повітряних Сил. - 2012. - № 3(32). - С. 134-136.

17. Galicki J., Czech M. A new approach to formulate the general strength theories for anisotropic discontinuous materials. Part A: The experimental base for a new approach to formulate the general strength theories for anisotropic materials on the basis of wood // Applied Mathematical Modelling. - 2013. - Vol. 37, no. 3. - P. 815-827. - DOI: 10.1016/j.apm.2012.03.004

18. Comparison of yield strength theories with experimental results / J. Niu, G. Liu, J. Tian, Y. Zhang and L. Meng // Engineering Mechanics. - 2014. - Vol. 31, no. 1. - P. 181-187. DOI: $10.6052 /$ j.issn.1000-4750.2012.09.0622

19. Liu G. A novel limiting strain energy strength theory // Transactions of Nonferrous Metals Society of China. - 2009. - Vol. 19, no. 6. - P. 1651-1662. - DOI: 10.1016/S1003-6326(09)60084-4

20. Deduction of geometrical approximation yield criterion and its application / S. Zhang, B. Song, X. Wang, D. Zhao and X. Chen // Journal of Mechanical Science and Technology. - 2014. Vol. 28, no. 6. - P. 2263-2271. - DOI: 10.1007/s12206-014-0515-6

21. Zhu X.-K., Leis B.N. Average shear stress yield criterion and its application to plastic collapse analysis of pipelines // International Journal of Pressure Vessels and Piping. - 2006. Vol. 83, no. 9. - P. 663-671. - DOI: 10.1016/j.ijpvp.2006.06.001

22. Kalnins A., Updike D.P. Limit Pressures of Cylindrical and Spherical Shells // Journal of Pressure Vessel Technology. - 2001. - Vol. 123, no. 3. - P. 288-292. - DOI: 10.1115/1.1367273

23. Zezin Y. P. Experimental investigation of the strength properties of particulate polymeric composites. - 2016. - Vol. 1785. - P. 030036. - DOI: 10.1063/1.4967057

24. Research on burst pressure for thin-walled elbow and spherical shell made of strength differential materials / L. Yan, Z. Junhai, X. Ergang, C. Xueye // Materials Research Innovations. 2015. - Vol. 19, no. 5. - P. 80-87. - DOI: 10.1179/1432891715Z.0000000001340

25. Shroff S., Kassapoglou C. Progressive failure modelling of impacted composite panels under compression // Journal of Reinforced Plastics and Composites. - 2015. - Vol. 34, no. 19. P. 1603-1614. - DOI: 10.1177/0731684415592485

26. Sengupta J., Ghosh A., Chakravorty D. Progressive Failure Analysis of Laminated Composite Cylindrical Shell Roofs // Journal of Failure Analysis and Prevention. - 2015. - Vol. 15, no. 3. - P. 390-400. - DOI: 10.1007/s11668-015-9951-6

27. Shokrieh M. M., Karamnejad A. Investigation of Strain Rate Effects on the Dynamic Response of a Glass/Epoxy Composite Plate Under Blast Loading by Using the Finite-Difference Method // Mechanics of Composite Materials. - 2014. - Vol. 50, no. 3. - P. 295-310. DOI: $10.1007 / \mathrm{s} 11029-014-9415-1$

28. Günel M., Kayran A. Non-linear progressive failure analysis of open-hole composite laminates under combined loading // Journal of Sandwich Structures \& Materials. - 2013. - Vol. 15, no. 3. - P. 309-339. - DOI: 10.1177/1099636213483651

29. Computational modeling of complex failure mechanisms in laminates / F. P. Van der Meer, L. J. Sluys, S. R. Hallett, M. R. Wisnom // Journal of Composite Materials. - 2012. - Vol. 46, no. 5. P. 603-623. - DOI: 10.1177/0021998311410473 
30. Pietropaoli E. Progressive Failure Analysis of Composite Structures Using a Constitutive Material Model (USERMAT) Developed and Implemented in ANSYS (C) // Applied Composite Materials. - 2012. - Vol. 19, nos. 3-4. - P. 657-668. - DOI: 10.1007/s10443-011-9220-0

31. Garnich M. R., Akula V. M. Review of Degradation Models for Progressive Failure Analysis of Fiber Reinforced Polymer Composites // Applied Mechanics Reviews. - 2009. - Vol. 62, no. 1. - P. 010801. - DOI: 10.1115/1.3013822

32. Bleyer J., De Buhan P. A numerical approach to the yield strength of shell structures // European Journal of Mechanics - A/Solids. - 2016. - Vol. 59. - P. 178-194. DOI: 10.1016/j.euromechsol.2016.03.002

33. Sun H.-H., Tan P.-L. Background of ABS Buckling Strength Assessment Criteria for Cylindrical Shells in Offshore Structures // Journal of Offshore Mechanics and Arctic Engineering. 2008. - Vol. 130, no. 2. - P. 021012. - DOI: 10.1115/1.2913349

34. Mellor P. B. The ultimate strength of thin-walled shells and circular diaphragms subjected to hydrostatic pressure // International Journal of Mechanical Sciences. - 1960. - Vol. 1, nos. 2-3. P. 216-228. - DOI: 10.1016/0020-7403(60)90041-2

35. Noh H. C. Ultimate strength of large scale reinforced concrete thin shell structures // ThinWalled Structures. - 2005. - Vol. 43, no. 9. - P. 1418-1443. - DOI: 10.1016/j.tws.2005.04.004

36. Zhang B., Sun Q. The Ultimate Strength of Stiffened Panel with Overall Buckling // Advanced Materials Research. - 2011. - Vol. 308-310. - P. 1297-1301. DOI: $10.4028 /$ www.scientific.net/AMR.308-310.1297

37. Ueda Y., Rashed S. M. H., Paik J. K. Buckling and ultimate strength interaction in plates and stiffened panels under combined inplane biaxial and shearing forces // Marine Structures. 1995. - Vol. 8, no. 1. - P. 1-36. - DOI: 10.1016/0951-8339(95)90663-F

38. Abrosimov N. A., Elesin A. V. Numerical analysis of dynamic strength of composite cylindrical shells under multiple-pulse exposures // PNRPU Mechanics Bulletin. - 2016. - No. 4. P. 7-19. - DOI: 10.15593/perm.mech/2016.4.01

39. Карпов В. В., Семенов А. А. Критерии прочности для тонкостенных ортотропных оболочек. Ч. 2. Расчеты и анализ // Вестник гражданских инженеров. - 2015. - № 1 (48). C. $60-70$.

40. Цветков С. В., Кулиш Г. Г. Критерии прочности однонаправленного органопластика при трехосном напряженном состоянии // Вестник МГТУ им. Н.Э. Баумана. Серия: Машиностроение. - 2011. - № SP. - C. 19-28.

41. $\mathrm{Yu} \mathrm{M.-H.} \mathrm{Advances} \mathrm{in} \mathrm{strength} \mathrm{theories} \mathrm{for} \mathrm{materials} \mathrm{under} \mathrm{complex} \mathrm{stress} \mathrm{state} \mathrm{the} \mathrm{20th}$ century // Appl. Mech. Rev. - 2002. - Vol. 55, no. 3. - P. 169-218. - DOI: 10.1115/1.1472455

42. Yu M.-H., Li J.-C. Computational plasticity: with emphasis on the application of the unified strength theory. - Hangzhou : Zhejiang Univ. Press, 2012. - 529 p.

43. Kolupaev V. A., Yu M.-H., Altenbach H. Visualization of the Unified Strength Theory // Archive of Applied Mechanics. - 2013. - Vol. 83, no. 7. - P. 1061-1085. - DOI: 10.1007/s00419013-0735-8

44. Цветков С. В. Критерии прочности трансверсально-изотропных материалов различных классов симметрии структуры // Известия ВУЗов. Машиностроение. - 2009. - № 1. C. $86-99$.

45. Карпов В. В., Семенов А. А. Критерии прочности для тонкостенных ортотропных оболочек. Ч. 1. Анализ основных критериев прочности изотропных и ортотропных материалов // Вестник гражданских инженеров. - 2014. - № 6 (47). - С. 43-51.

46. Смердов А. А., Буянов И. А., Чуднов И. В. Анализ оптимальных сочетаний требований к разрабатываемым углепластикам для крупногабаритных ракетно-космических конструкций // Известия высших учебных заведений. Машиностроение. - 2012. - № 8. C. $70-77$. 
47. Тышкевич В. Н. Выбор критерия прочности для труб из армированных пластиков // Известия Волгоградского государственного технического университета. - 2011. - № 5 (78). C. $76-79$.

48. Karpov V. V., Semenov A. A. Mathematical models and algorithms for studying strength and stability of shell structures // Journal of Applied and Industrial Mathematics. - 2017. - Vol. 11, no. 1. - P. 70-81. - DOI: 10.1134/S1990478917010082

49. Kuznetsov E. B. Continuation of solutions in multiparameter approximation of curves and surfaces // Computational Mathematics and Mathematical Physics. - 2012. - Vol. 52, no. 8. P. 1149-1162. - DOI: 10.1134/S0965542512080076 